\title{
Clinical evaluation of HPV DNA test combined with liquid-based cytology in the diagnosis of cervical disease ${ }^{*}$
}

\author{
Raghvendra Thakur ${ }^{1}$, Jasma Mally ${ }^{2}$, Rajina Shrestha ${ }^{3}$, Xuehua Zheng ${ }^{4}$, Mengli Zhang ${ }^{1}$, Yanjun Wu ${ }^{1}$, \\ Neelima Bajracharya', Guiying Zheng ${ }^{1 \#}$ \\ ${ }^{1}$ Department of Obstetrics and Gynecology, Second Hospital of Jilin University, Norman Bethune College of Medicine, Changchun, \\ China \\ ${ }^{2}$ Department of Obstetrics and Gynecology, First Hospital of Jilin University, Norman Bethune College of Medicine, Changchun, \\ China \\ ${ }^{3}$ Department of Obstetrics and Gynecology, Jiamusi University, Jiamusi, China \\ ${ }^{4}$ Department of Obstetrics and Gynecology, Maternity and Children Hospital of Jilin Province, Liaoyuan, China \\ Email: thakurrags@hotmail.com, jasma.mally@yahoo.com, rajinashrestha76@yahoo.com,xuehuazheng3@gmail.com, \\ zhml851202@126.com, wuyanjunsd7@163.com, neelimabajracharya@gmail.com, "\#lyzzgy99@163.com
}

Received 22 March 2013; revised 21 April 2013; accepted 28 April 2013

Copyright (c) 2013 Raghvendra Thakur et al. This is an open access article distributed under the Creative Commons Attribution License, which permits unrestricted use, distribution, and reproduction in any medium, provided the original work is properly cited.

\begin{abstract}
Objectives: To evaluate the sensitivity and accuracy of the HPV DNA test in conjunction with thin prep cytology test as a screening method of human papillomavirus (HPV) infection. To study either the cervical erosion is related to high risk HPV infection or to determine the mean age distribution that is more prone to HPV infection. Material and Methods: The study is a retrospective cohort implemented to determine the real performance of liquid based medium and HPV DNA testing combined in second clinical hospital of Jilin University Changchun, China. The study group included total 150 patients from January 1, 2011 to December 30, 2012. A computerized search identified patients with thin prep test results and high risk HPV DNA testing during a 2-year period was recruited. The patients were chosen after proper speculum examination followed by thin prep cytology (TCT) and HPV DNA test. Cytologic specimens were obtained with endocervical brush, which was rinsed into the vial of Cytyc. The residual samples after the cytology report were taken for reflex HPV DNA test. The manufacture protocol was followed for HPV DNA testing using Hybrid Capture II. Colposcopic biopsy was performed for the diagnosis purpose, in patients who had atypical squamous cells of undeter-

\footnotetext{
*Authors' contributions: Thakur, R. and Mally, J. designed the study and carried out most of the study; all authors collected the clinical information and provided valuable suggestions in the preparation of the manuscript.

Competing interests: The authors declare that they have no competing interests.

\#Corresponding author.
}

mined significance (AUS-US), low grade intraepithelial lesion (LSIL) or high-grade intraepithelial lesion (HSIL) in cytology and with positive results of highrisk HPV DNA. The diagnostic criteria were based on the Bethesda System (TBS). Findings: The high risk HPV positive women with abnormal cytology had a CIN I risk of $73(86 \%)$, whereas $35(23.3 \%)$ high-risk HPV positive women out of 109 (72.7\%) normal cytology who underwent histological biopsy had CIN I $16(10.7 \%)$. The risk for cervical intraepithelial neoplasia (CIN) in women with high-risk HPV positive with normal cytology was higher among women invited for the first time 31 - 40 years of age $12(8 \%)$ than among older women $1(0.7 \%)$. Out of $44(29.3 \%)$ women who had I degree erosion with $6(14 \%)$ positive HPV DNA test $38(86 \%)$ had a normal histology biopsy showing no statically significant between them. Conclusion: The data confirm that HR-HPV DNA testing is much more sensitive than cytology alone and that HPV DNA testing helps in identifying women with high risk of serious cervical disease in an efficient and medically acceptable manner. The other most significant advantage of this cervical cancer screening method is that women who are HPV DNA positive can easily and quickly referred for colposcopic examination (within one year), which could identify the precancerous and cancer stage. And those who are HPV DNA negative can safely have much longer screening intervals saving considerable costs. With mean age being $38 \pm 10$ years, age older than 30 years should undergo HPV DNA testing with cytology triage in primary screening. But in woman younger 
than 30 years using HPV DNA assay, as an initial screening step can increase the prevalence of abnormal smears and the positive predictive value of HPV followed by TCT. However, close follow-up is essential if the initial biopsy is negative because a considerable number of women may have HPV infection positive in subsequent studies.

Keywords: Human Papillomavirus; Thin-Prep Cytology Test; Hybrid Capture; Cervical Intraepithelial Neoplasia; Bethesda System; Colposcopy Examination

\section{INTRODUCTION}

Human papilloma virus (HPVs) being the most common sexually transmitted infection worldwide is studied and taken more interest. Papillomaviruses belongs to the Papilloma viridae family. More than 40 types of HPV are identified which can transmit through sexual contact. These are DNA viruses and targets stratified squamous epithelia of the body. A subset is also able to infect the grandular epithelia of the cervix. HPVs are distributed throughout the body but with different anatomic predilections, which allows to distinguished three major groups: 1) the HPV types found in cutaneous warts; 2) those associated with epidermodysplasia verruciformis; and 3) the HPVs associated genital or mucosal lesion. Depending upon the HPV types may cause genital warts or "high risk "HPV may progress to precancerous lesion and invasive cancer [1]. Among all the HPV genotypes, types 6 and 11 are predominantly responsible for the benign diseases, and types 16 and 18 are strongly associated with oropharynx and cervical cancer [2].

HPV lesions are thought to arise from the proliferation of infected basal keratinocytes. It is limited to basal cells of stratified epithelium, which could be exposes to infectious virus through disturbed epithelial barrier as might occur after minor skin abrasions or sexual intercourse.

Most of HPV infections in young females are only temporary and are gone in 1 - 2-year duration [3], but some high risk viruses may take 10 - 15 years to progress into invasive cervical cancer. Genital HPV infections cause significant health burden, because cervical cancer is the second most common and lethal cancer detected in women worldwide. Progression to cervical cancer can be almost always prevented when standard prevention strategies are applied.

Preventing HPV before it reaches the disease stage remains challenge. There has been clinical significant effectiveness of male condom in preventing genital HPV infections and disease. However, two vaccines are now available in many countries since June 2006, both based on the concept of virus- like particles (VLPs). The first one is Gardasil, is directed against HPV types 6 and 11, which account for $80 \%$ of external genital warts, whereas the other is Cervarix, is directed against only HPV 16 and 18 which is responsible for $70 \%$ of cervical cancer.

Various screening and treatment modalities have been proposed in recent years. Papanicaloau (Pap) smear or liquid-based cytology is widely used to detect abnormal cells, though Pap smear has decreased the incidence if cervical cancer but even so, the mortality in resource poor areas. Worldwide, there are estimated to be 490,000 cases and 270,000 deaths each year [4,5]. However, screening programs incur considerable financial and psychosocial costs [6,7].

\section{MATERIAL AND METHODS}

A retrospective cohort study was designed for the study purpose. A computerized search identified patients with thin-cytology test results and high risk HPV DNA testing during a 2-year period, January 1, 2011, to December 30, 2012. The study group included 150 patients from Gynecology outpatient department of Norman Bethune College of Medicine, second clinical Hospital, Jilin University, Changchun, China.

The patients were chosen with a proper speculum examination followed by Thin-cytology test and HPV DNA test. Patients were recruited with either positive or negative Thin- cytology test with HPV DNA test. The erosive cervix in speculum examination was also tested for HPV DNA test. A threshold of $1 \mathrm{pg} / \mathrm{ml}$ of HPV DNA was considered a positive result and recommended for colposcopic directed biopsy to confirm the diagnosis. The medical records were reviewed.

Cytology specimens were obtained with endocervical brush, which was rinsed into the vial of Cytyc. The residual samples after the cytology report issued were taken for reflex HPV DNA test. The manufacturer's protocol was followed for HPV DNA testing using the Hybrid Capture II. The double- stranded DNA is denatured into single-stranded DNA and combined with RNA probes. If HPV DNA is present in the specimen the DNA-RNA complexes or hybrids are formed and immobilized by antibodies present in the capture tube. When an enzyme-linked antibody is added to the capture tube, it has ability of binding the immobilized DNA-RNA hybrids into multiple sites. The presence of HPV DNA is detected after adding a chemiluminescent substrate. In accordance with the ASCCP guidelines, only high-risk types $(16,18,31,33,35,39,45,51,52,56,58,59$, and 68) were tested.

\subsection{Inclusion Criteria}

For the study purpose, the patients were selected who had:

Proper Medical Record; 
Age groups are included more than 20 years;

Patients who have gone through the examination for the first time;

A patient who had abnormal either in the speculum examination, thin-cytology test or HPV DNA test.

\subsection{The Exclusion Criteria}

1) Insignificant follow-up and medical records;

2) Patients with normal thin-cytology and HPV DNA test;

3) Follow-up examination tests.

\subsection{Data Collection Tools}

The contents of the intended queries were mentioned sequentially. The data were collected and filled in the respected columns.

\subsection{Study Variables}

- Age;

- Liquid based Cytology Test (TCT);

- Atypical Squamous Cells of Undermined Significance (ASC-US);

- HPV DNA Test;

- Colposcopic directed biopsy.

\subsection{Statistical Analysis}

All tests of significance were 2 tailed and p values of $<0.05$ was considered as statistically significant. The statistical analysis, are performed using SPSS, 19.0 Window version, statistical package.

The sensitivity, specificity, positive predictive value and negative value were evaluated with the thin-cytology test and HPV DNA tests.

The sensitivity is equal to the number of true positives divided by the total number of true positives plus the number of false negatives.

The specificity is equal to the number of true negative divided by the number of true negative plus false positive.

The positive predictive value is equal to the number of true positive divide by the number of rue positive plus false positive.

The negative predictive value is equal to the number of true negative divided by the number of true negative plus false negative.

\section{RESULTS}

Thin-cytology test reviewed in 109 (72.7\%) patient which shows normal results, 13 (8.7\%) had atypical squamous cells of undetermined origin (ASC-US), 14 (9.3\%) had a low squamous intraepithelial lesion (LSIL) and 14 (9.3\%) had a high squamous intraepithelial lesion
(Table 1, Figure 1).

Our study demonstrates the clinical distribution of the degrees of erosion in speculum examination. The total number of patients who had, 1st degree erosion in speculum examination were 44 (29.3\%), 2nd degree erosions were $40(26.7 \%)$ and 3rd degree erosions were (2.7\%). With HPV DNA load threshold of $1 \mathrm{pg} / \mathrm{ml}$ considering to be positive in $29.3 \%$ I degree erosion 38 (86\%) had normal results showing there is no statistically significant relation between HPV infection and degree of erosions $(\mathrm{P}>0.05)$ (Table 2, Figure 2).

The percentage of patients who underwent histological examination after atypical squamous cells of undetermined origin was diagnosed in liquid based cytology. 13 (8.7\%) were diagnosed as ASC-US in the thin-cytology test. Of these patients, $1(0.7 \%)$ had high-risk HPV DNA identified by Hybrid Capture II and all went through

Table 1. Distribution of clinically significant findings of thincytology test review of 150 patients*.

\begin{tabular}{cc}
\hline Thin-Cytology Test (TCT) & Patient (\%) \\
\hline Normal & $109(72.7 \%)$ \\
ASC-US & $13(8.7 \%)$ \\
Low-grade dysplasia & $14(9.3 \%)$ \\
High-grade dysplasia & $14(9.3 \%)$ \\
Total & $150(100 \%)$ \\
\hline
\end{tabular}

ASC-US: Atypical squamous cells of undetermined significance; ${ }^{*}$ Data are given as number (percentage).

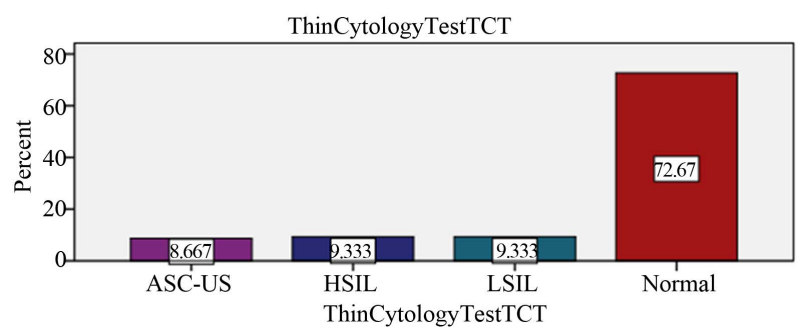

Figure 1. Clinical evaluation of the results in the liquid based medium.

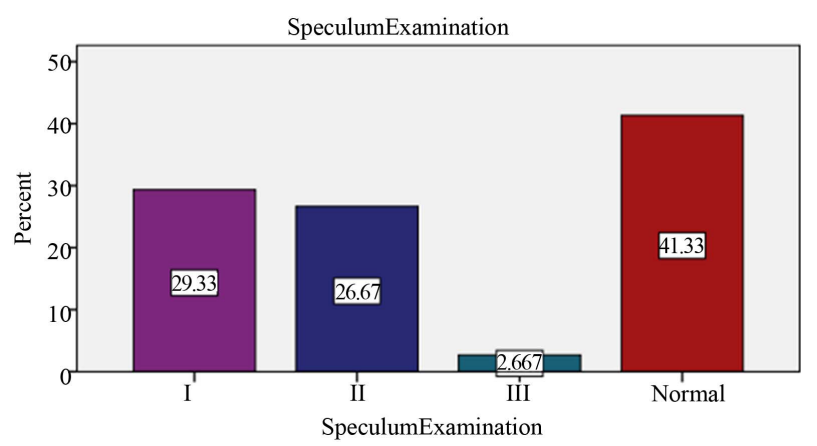

Figure 2. Degree of erosion in Speculum Examination. 
Table 2. Distribution of clinically significant degrees of erosions in speculum examination of 150 patients* .

\begin{tabular}{cc}
\hline Speculum Examination & Patient (\%) \\
\hline Normal & $62(41.3 \%)$ \\
I degree erosion & $44(29.3 \%)$ \\
II degree erosion & $40(26.7 \%)$ \\
III degree erosion & $4(2.7 \%)$ \\
Total & $150(100 \%)$ \\
\hline
\end{tabular}

*Data are given in number (percentage).

histological examination. Overall, 13 (8.7\%) of patient with ASC-US interpretation underwent histological follow-up and 8 (5.3\%) has cervical intraepithelial neoplasia (CINI) positive whereas, 14.2 (94.7\%) had CIN negative results. It shows the relationship between ASC-US and HPV DNA load is non-significant $(\mathrm{P}>0.05)$ (Table 3).

Study shows the variation in the liquid based cytology and HPV DNA testing of total patients. 109 (72.7\%) patients had a normal thin-cytology test (TCT) results. 72.7\% underwent HPV DNA testing who had some degree of erosions in speculum examination to rule out the HPV infection and further histological examination if HPV DNA load was higher than the average. Out of 109 (72.7\%) normal TCT results, the HPV DNA test positive were 35 (23.3\%) and cervical intraepithelial neoplasia (CIN I) was found to be in 16 (10.7\%). The significance of the combined test of TCT and HPV DNA test were specifically high $(\mathrm{P}<0.05)$ than alone (Table 4$)$.

Regarding age-specific distribution of human papillomavirus infection in Changchun, the mean age distribution who was more prone to HPV infection were, $37.8 \pm$ 10.44. At age 20 - 30 years 40 (26.7\%), 5 (3.3\%) had cervicitis and $4(2.7 \%)$ had CIN I. At age 31 - 40 years 50 (33.3\%), 5 (3.3\%) had cervicitis and 12 (8\%) had CIN I and $1(0.7 \%)$ had cervical carcinoma. At age $41-50$ years 46 (30.7\%), 2 (1.3\%) had cervicitis, 8 (5.3\%) had CIN I and $1(0.7 \%)$ had cervical carcinoma. Following age 51 - 60 years 10 (6.7\%), 1 (0.7\%) had cervicitis, 2 $(1.3 \%)$ had CIN I. And at age $61-70$ years, $0(0 \%)$ had cervicitis, $1(0.7 \%)$ had CIN I and $1(0.7 \%)$ had cervical carcinoma. These age variation shows decline in cervicitis and inclined in cervical carcinoma. The risk for CIN is higher among women 31 - 40 years of age 12 (8\%) than the older women 1 (0.7\%) (Table 5, Figure 3).

\section{DISCUSSION}

Human papillomavirus (HPV) has clearly shown to be the cause of most cervical cancers. Given that, lots of interest is growing worldwide in the potential for use of HPV diagnostic on cervical cancer prevention programs,
Table 3. Distribution of clinically significant of atypical squamous cells of undermined significance with cervical intraepithelial neoplasia of 150 patients*.

\begin{tabular}{rc}
\hline & Patient (\%) \\
\hline ACS-US & $13(8.7 \%)$ \\
CIN Positive & $8(5.3 \%)$ \\
CIN Negative & $14.2(94.7 \%)$ \\
\hline
\end{tabular}

ASC-US: Atypical squamous cells of undetermined significance; CIN: Cervical intraepithelial neoplasia; ${ }^{*}$ Data are given in number (percentage).

Table 4. Distribution of clinically significant combination of normal thin-cytology test with HPV-DNA positive testing of 150 patients ${ }^{*}$.

\begin{tabular}{cc}
\hline & Patient (\%) \\
\hline TCT normal & $109(72.7 \%)$ \\
HPV DNA positive & $35(23.3 \%)$ \\
CIN I & $16(10.7 \%)$ \\
\hline
\end{tabular}

TCT: thin prep cytology test; HPV DNA testing is done with the hybrid capture II; CIN: Cervical intraepithelial neoplasia; "Data are given in number (percentage).

Table 5. Age specific distribution of human papilloma virus infection in Changchun*.

\begin{tabular}{cccccc}
\hline Age & $20-30 \mathrm{yrs}$ & $31-40 \mathrm{yrs}$ & $41-50 \mathrm{yrs}$ & 51 - $60 \mathrm{yrs}$ & $61-70 \mathrm{yrs}$ \\
\hline & $\mathrm{n}=40$ & $\mathrm{n}=50$ & $\mathrm{n}=46$ & $\mathrm{n}=10$ & $\mathrm{n}=4$ \\
& $(26.7 \%)$ & $(33.3 \%)$ & $(30.7 \%)$ & $(6.7 \%$ & $(27 \%)$ \\
\hline Cervicitis & $5(3.3 \%)$ & $5(3.3 \%)$ & $2(1.3 \%)$ & $1(0.7 \%)$ & $0(0 \%)$ \\
CIN I & $4(2.7 \%)$ & $12(8 \%)$ & $8(5.3 \%)$ & $2(1.3 \%)$ & $1(0.7 \%)$ \\
CIN II & $2(1.3 \%)$ & $2(1.3 \%)$ & $3(2 \%)$ & $3(2 \%)$ & $0(0 \%)$ \\
CIN III & $2(1.3 \%)$ & $4(2.7 \%)$ & $6(4 \%)$ & $0(0 \%)$ & $1(0.7 \%)$ \\
\hline
\end{tabular}

CIN: Cervical intraepithelial neoplasia; The mean age distribution is $37.8 \pm$ 10.44 years. ${ }^{*}$ Data are given in number (percentage).

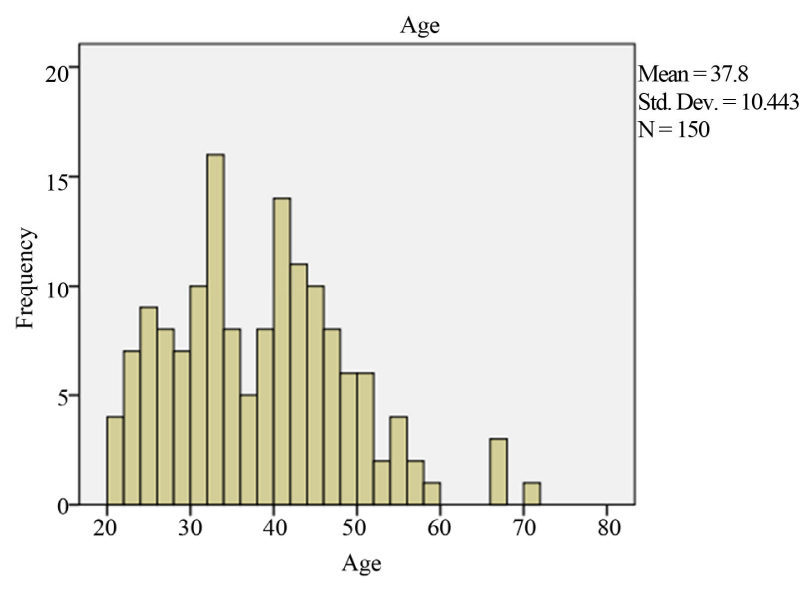

Figure 3. Age specific distribution. 
both as an adjunct to cytological screening and in primary screening for cervical dysplasia. Many efforts have been made to develop methods, which would enhance the sensitivity and specificity of the Papanicolaou smear. From various researches the evolution of liquid-based gynecologic specimen was resulted. The researchers argue that liquid-based preparations outperform conventional method smears because of improved fixation; standardizations of cell transfer and decreased obscuring factors. Another advantage of liquid based cytology is that the transport media containing the remaining cells could be stored and used for HPV DNA testing. The researchers also point out that, in direct smears, the cells may not transfer in a representative way and that up to $90 \%$ of the scraped materials from the cervix may be discarded. As with liquid-based collection, the sampling will be operator dependent and variation is very less likely, since the laboratory controls the processing. And with proper sampling liquid based cytology has the advantage of being suitable for high-risk HPV testing and could reduce unsatisfactory specimens from $4.1 \%$ to $2.6 \%[8]$.

According to the studies, the accuracy of conventional cytology report is sensitivity $72 \%$ and specificity $94 \%$ whereas, liquid based monolayer cytology report is sensitivity $61 \%$ to $66 \%$ and specificity of $82 \%$ to $91 \%$ [9]. Various methods of liquid based medium have been introduced and the US FDA for cervico-vaginal testing currently approves Sure Path and Thin Prep 2000 System. However, due to the high cost and the requirement of Cytotechnologist and a pathologist to interpret the result has led to the development and evaluation of other alternative and less costly methods. Khalbuss et al. (2000) introduced Spin Thin method, and its results correlated very well with conventional smears and follow- up histology. Likewise, Johnson, et al. (2000) used cervical collection device into $15 \mathrm{ml}$ of Cyto Rich Red, a proprietary formula of buffering agents, emulsifiers, formaldehyde and alcohol. The main benefit of this method was reusability of its funnel assembly, which reduced the bulk of disposable plastics, which could impact the environment and add the cost.

HPV DNA has gained lots of attention due to its higher sensitivity and detection of "high-risk" HPV types that most commonly affect the cervix. These tests detect DNA from 18 most prevalent types of HPV that affect the cervix, 13 "high-risk" and 5 "low-risk". The high and low-risk testing is done separately and the test does not specify the type of HPV involved [10]. According to the National Cancer Institute, HPV DNA test is an effective way to identify the high-risk HPV. And, the FDA has approved an HPV test as a follow-up for women who have an ambiguous cytology test and for woman more than 30 years of age, as a general cervical screening.
FDA commercially approved and made the product available for testing HPV DNA, the Hybrid Capture II by Qiagen [11]. Although high-risk HPV DNA testing using Hybrid Capture II assay is more objective than cytology test, legitimate false-positive HPV DNA test results have been encountered [12]. Though, HPV testing has shown higher sensitivity, but it's uncertain either the highest sensitivity is dependent on the age of the woman being screened. The studies have shown that, the presence of a very high load of low-risk HPV DNA in the sample can cross-hybridize with high-risk HPV DNA probes leading to the false-positive results for high-risk subtypes. The incidence of false-positive is very low, less than $2 \%$ and is dependent on the cutoff value [13, 14].

In the present study population, there were a substantial number of atypical squamous cells of undetermined significance (ASC-US) cases with a positive HPV DNA test result and a negative biopsy result, supporting the validity of the negative biopsy diagnosis. The studies have proved that the HPV infections can be transient and episodic, especially if the woman is young $[15,16]$. If the HPV infection is newly acquired the chance to resolve is higher than the longer persistent infection. The study shows that, the probability of resolving the newly acquired infection is $31 \%$ in the first 6-month's period and $39 \%$ during second period [17]. However, with the time period the resolution of infection drops $11 \%$ in the third 3-month's period after infection [18]. Even in our studies, it is reasonable to assume that some newly acquired HPV infections might have resolved even before they underwent colposcopic examination and biopsy resulting in false-positive HPV DNA testing. In the study, if only the cases of "negative for dysplasia" to "high-grade dysplasia" were considered, $10.7 \%$ of the patients had an upgrade in their diagnosis after the additional biopsy examination. This significant number is enough to believe that recommending an additional biopsy examination in HPV positive patients with positive cytology findings for intraepithelial lesion.

According to the observation $14 \%$ of the patients who were HPV DNA test result positive had a negative biopsy result. Almost one third of the cases had clinically significant lesions when additional examinations were done. With this, we may conclude that additional levels if there is a discrepancy between the HPV DNA test result and the cytological findings. However, close followup is essential if the initial biopsy result is negative because a considerable number of women may have HPV infection positive in subsequent studies.

\section{CONCLUSION}

The data from this study confirms that HR-HPV DNA testing is much more sensitive than cytology alone and 
that HPV DNA testing helps in identifying women with high risk of serious cervical disease in an efficient and medically acceptable manner. The other most significant advantage of this cervical cancer screening method is that women who are HPV DNA positive can easily and quickly referred for colposcopic examination (within one year), which could identify the precancerous and cancer stage. And those who are HPV DNA negative can safely have much longer screening intervals saving considerable costs. With mean age being $38 \pm 10$ years, age older than 30 years should undergo HPV DNA testing with cytology triage in primary screening. But in woman younger than 30 years using HPV DNA assay, as an initial screening step can increase the prevalence of abnormal smears and the positive predictive value of HPV followed by TCT. However, close follow-up is essential if the initial biopsy is negative because a considerable number of women may have HPV infection positive in subsequent studies.

\section{ACKNOWLEDGEMENTS}

The authors would like to thank Prof. Guiying Zheng for her valuable second opinion on manuscript.

\section{REFERENCES}

[1] Schiffman, M. and Castle, P.E. (2003) Human papillomavirus: Epidemiology and public health. Archives of Pathology \& Laboratory Medicine, 127, 930-934.

[2] Gillison, M.L. (2004) Human papillomavirus-associated head and neck cancer is a distinct epidemiologic, clinical, and molecular entity. Seminars in Oncology, 31, 744-754. doi:10.1053/j.seminoncol.2004.09.011

[3] Goldstein, M.A., Goodman, A., Del Carmen, M.G. and Wilbur, D.C. (2009) Case records of the Massachusetts General Hospital. Case 10-2009. New England Journal of Medicine, 360, 1337-1344.

[4] Kahn, J.A. (2009) HPV vaccination for the prevention of cervical intraepithelial neoplasia. New England Journal of Medicine, 361, 271-278. doi:10.1056/NEJMct0806938

[5] NCCC National Cervical Cancer Coalition, 218-226. Retrieved 1 July 2008.

www.nccn.org/professionals/physician_gls/f_guidelines.a sp\#cervical

[6] Peto, J., Gilham, C., Fletcher, O. and Matthews, F.E. (2004) The cervical cancer epidemic that screening has prevented in the UK. Lancet, 364, 249-256. doi:10.1016/S0140-6736(04)16674-9

[7] Solomon, D., Schiffman, M., Tarone, R. and The ALTS
Study Group (2001) Comparison of three management strategies for patients with atypical squamous cells of undetermined significance: Baseline results from a randomized trial. Journal of the National Cancer Institute, 93, 293-299. doi:10.1093/jnci/93.4.293

[8] Ronco, G., Cuzick, J., Pierotti, P., et al. (2007) Accuracy of liquid based versus conventional cytology: Overall results of new technologies for cervical cancer screening randomized controlled trial. British Medical Journal, 335, 28. doi:10.1136/bmj.39196.740995.BE

[9] Coste, J., Cochand-Priollet, B., De Cremoux, P., et al. (2003) Cross sectional study of conventional cervical smear, monolayer cytology, and human papillomavirus DNA testing for cervical cancer screening. British Medical Journal, 326, 733. doi:10.1136/bmj.326.7392.733

[10] Tristram, A. and Fiander, A. (2007) Human papillomavirus (including vaccines). Obstetrics, Gynaecology and Reproductive Medicine, 17, 324-329. doi:10.1016/j.ogrm.2007.08.006

[11] Davies, P., Kornegay, J. and Iftner, T. (2001) Current methods of testing for human papillomavirus. Best Practice and Research in Clinical Obstetrics and Gynaecology, 15, 677-700. doi:10.1053/beog.2001.0214

[12] De Cremoux, P., Coste, J., Sastre-Garau, X., et al. (2003) Efficiency of the Hybrid Capture 2 HPV DNA test in cervical cancer screening: A study by the French Society of Clinical Cytology. American Journal of Clinical Pathology, 120, 492-499. doi:10.1309/XFUCPP6M5XUA94B8

[13] Klinkhamer, P.J., Vooijs, G.P. and De Haan, A.F. (1988) Intraobserver and interobserver variability in the diagnosis of epithelial abnormalities in cervical smears. Acta Cytologica, 32, 794-800.

[14] Parker, M.F., Zahn, C.M., Vogel, K.M., et al. (2002) Discrepancy in the interpretation of cervical histology by gynecologic pathologists. Obstetrics \& Gynecology, 100, 277-280. doi:10.1016/S0029-7844(02)02058-6

[15] Ho, G.Y., Bierman, R., Beardsley, L., et al. (1998) Natural history of cervicovaginal papillomavirus infection in young women. The New England Journal of Medicine, 338, 423-428. doi:10.1056/NEJM199802123380703

[16] Woodman, C.B., Collins, S., Winter, H., et al. (2001) Natural history of cervical human papillomavirus infection in young women: A longitudinal cohort study. Lancet, 357, 1831-1836. doi:10.1016/S0140-6736(00)04956-4

[17] Richart, R.M. and Barron, B.A. (1969) A follow-up study of patients with cervical dysplasia. American Journal of Obstetrics and Gynecology, 105, 386-393.

[18] Nasiell, K., Nasiell, M. and Vaclavinkova, A. (1983) Behavior of moderate cervical dysplasia during long-term follow-up. Obstetrics and Gynecology, 61, 609-614. 